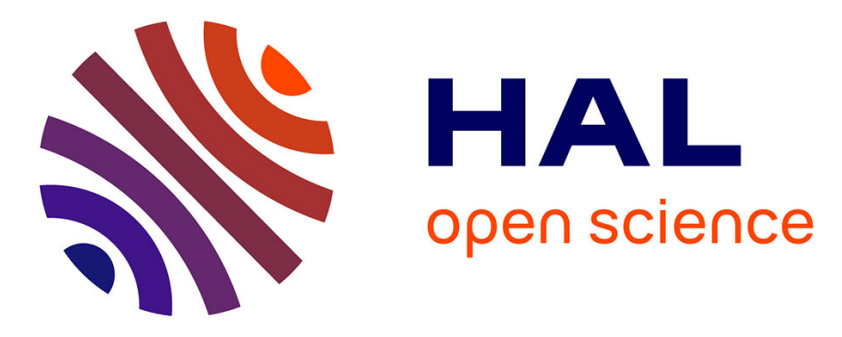

\title{
Le traitement archéologique des vestiges osseux animaux : compte-rendu de la table ronde
}

Dominique Joly, Lucien Jourdan, Patrice Méniel, François Poplin, Marc

Turquet, J.-D. Vigne

\section{To cite this version:}

Dominique Joly, Lucien Jourdan, Patrice Méniel, François Poplin, Marc Turquet, et al.. Le traitement archéologique des vestiges osseux animaux: compte-rendu de la table ronde. Revue archéologique de Picardie, 1983, Les Celtes dans le nord du bassin parisien. Actes du Ve colloque de l'Association française pour l'étude de l'âge du Fer, 1, pp.298-301. 10.3406/pica.1983.3013 . hal-02536334

\section{HAL Id: hal-02536334 \\ https://hal.science/hal-02536334}

Submitted on 8 Apr 2020

HAL is a multi-disciplinary open access archive for the deposit and dissemination of scientific research documents, whether they are published or not. The documents may come from teaching and research institutions in France or abroad, or from public or private research centers.
L'archive ouverte pluridisciplinaire HAL, est destinée au dépôt et à la diffusion de documents scientifiques de niveau recherche, publiés ou non, émanant des établissements d'enseignement et de recherche français ou étrangers, des laboratoires publics ou privés.

\section{(1) (1) $\$$}

Distributed under a Creative Commons Attribution - NonCommercial - NoDerivatives| 4.0 


\title{
LE TRAITEMENT ARCHÉOLOGIQUE DES VESTIGES OSSEUX ANIMAUX
}

\author{
COMPTE RENDU DE LA TABLE RONDE *
}

En réponse aux vœux exprimés par beaucoup d'archéologues, nous nous sommes proposés, à l'occasion du colloque sur "le deuxième Age du Fer dans l'Oise et le Nord de la Région parisienne", de présenter les informations nécessaires quant au traitement des vestiges osseux. Bien que certains soient déjà largement informés sur ce sujet, l'expérience prouve que cette démarche n'est pas totalement inutile.

Nous ne présentons donc pas ici de résultats d'étude de faune. Il s'agit de remarques méthodologiques, parmi lesquelles nous privilégions les aspects techniques. Mais le lecteur doit garder présent à l'esprit qu'il n'existe pas de recette universelle en la matière. L'objectif est de préserver l'information transmise par les vestiges osseux, ce qui nécessite qu'on se soumette à un certain nombre de contraintes. C'est au fouilleur d'adapter les méthodes aux conditions archéologiques et aux contingences matérielles.

\section{PRÉlèvement des ossements animauX}

L'histoire taphonomique du matériel osseux est marquée par trois étapes :

- la fragmentation et la disparition de certains vestiges avant l'enfouissement, essentiellement par l'intervention de l'homme et de ses animaux domestiques :

- l'enfouissement, à l'origine de la destruction différentielle ;

- la fouille et les étapes ultérieures du traitement archéologique qui entraînent, elles aussi, une perte d'informations.

Les conditions de fouille, de relevé et de préparation des ossements animaux sont les seúles étapes de ce processus que l'archéologue puisse contrôler et il convient, dans la mesure du possible, de limiter leurs effets parasites.

Tout d'abord, on doit s'efforcer de prélever la totalité des vestiges osseux.

Le dégagement et le prélèvement : ils doivent être réalisés de manière à ne pas modifier l'état initial des vestiges. En particulier, il faut éviter de produire

\footnotetext{
* Dominique JOLY : Archéologue

Mairie de Chartres, 28000 CHARTRES

Lucien JOURDAN : Centre de Recherches Archéologiques

Laboratoire d'Ostéologie Sophia Antipolis

05560 VALBONNE

Patrice MENIEL : CRAVO, 21 rue des Cordeliers,

60200 COMPIĖGNE

François POPLIN : Museum National d'Histoire Naturelle

Laboratoire d'Anatomie Comparée, 55 rue Buffon

75005 PARIS

Marc TURQUET : 23 rue des Quatre Vents

92380 GARCHES

Jean-Denis VIGNE : c/o F. Poplin

Museum National d'Histoire Naturelle

Laboratoire d'Anatomie Comparée, 55 rue Buffon
}

sur les surfaces osseuses des traces supplémentaires à celles éventuellement présentes. S'il est possible de dégager un os avec une truelle sans altérer sa surface, l'opération est évidemment plus facile avec un instrument en bois.

Le prélèvement ne doit pas être source de fractures supplémentaires. Dans la plupart des cas, un dégagement minutieux suffit, mais quand les caractéristiques physico-chimiques des vestiges sont mauvaises, une consolidation durant la fouille est nécessaire. II existe plusieurs produits pour renforcer les ossements fragiles :

- la colle de menuisier, diluée dans l'eau ;

- le rhodopas. Sous ce nom sont regroupés des produits de Rhône-Poulenc qui peuvent se dissoudre dans des solvants divers : acétone, alcool, xylène, ...;

- I'acétate de cellulose dissout dans l'acétone, que l'on peut remplacer par de la colle cellulosique, type colle Scotch.

Ces dernières solutions sont très efficaces car leur solvant a un grand pouvoir mouillant et imprègne profondément l'os en en chassant toute trace d'humidité.

La solution doit donc être suffisamment fluide pour bien s'infiltrer dans l'os. Pour ceci, on ne doit pas avoir l'impression que la fluidité du solvant a été modifiée par l'adjonction de colle ou d'acétate, ce qui correspond à une dilution d'environ $10 \%$.

Les crânes doivent faire l'objet de soins particuliers du fait de leur intérêt zoologique. II faut les prélever sans les briser et leur assurer de bonnes conditions de transport en les calant soigneusement dans des boites rigides. Un crâne entier peut être nettoyé et consolidé en peu de temps, alors qu'un crâne brisé doit faire l'objet d'une restauration longue (plusieurs dizaines d'heures). Il convient d'autre part de prélever et de tamiser le sédiment voisin du crâne de façon à récupérer certaines parties détachables (dents, hyoïdes, ...).

On découvre parfois des ossements en connexion anatomique. Ces pièces, très importantes pour l'interprétation paléo-ethnographique et paléo-zoologique, nécessitent des précautions supplémentaires. II faut fouiller le plus complètement possible ces ensembles avant d'entreprendre leur "démontage", de façon à avoir une vision globale et un enregistrement exhaustif. La fouille complète permet de "suivre la structure anatomique", de la comprendre, et d'éviter ainsi les pertes (de relation ou d'os). Dans ce cas, un minimum de connaissances anatomiques peut se révéler d'un grand secours.

Le relevé des ossements : il doit faire l'objet des mêmes soins que celui des autres vestiges. II faut rappeler, en effet, que les os peuvent faire plus facilement l'objet de remontages (collage, connexion, appariements, ...) et nous renseignent ainsi sur l'organisation de la structure. 
Le tamisage : il est nécessaire ; il peut, en effet, apporter un complément d'information qui permet de corriger les proportions calculées sur le nombre de restes, mais aussi d'allonger la liste des espèces présentes, notamment en ce qui concerne la microfaune. Les modifications apportées par un tamisage sur un échantillon provenant d'une fouille archéologique, ont été illustrées par S. PAYNE (fig. 1). II ne faut pas oublier par ailleurs, que ce procédé permet, par la même occasion, de récupérer les graines, les coquillages, etc.

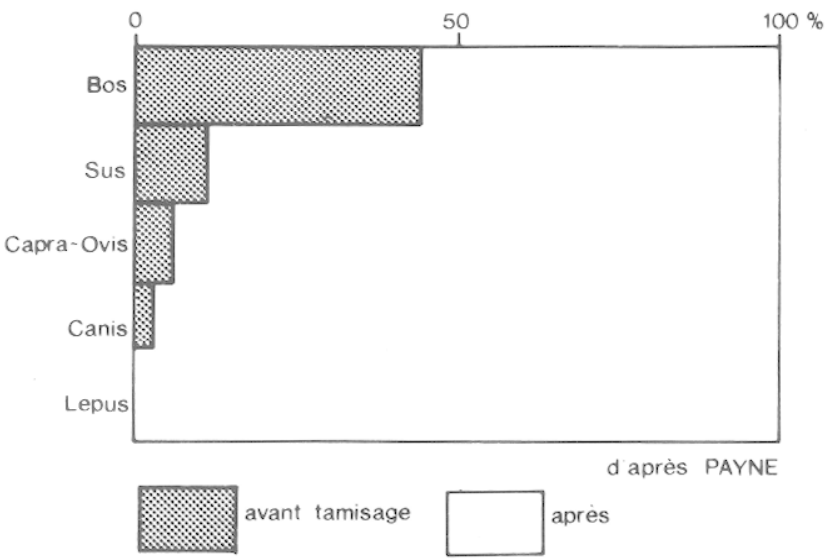

Fig. 1 : d'après S. PAYNE. Partial recovery and sample bias, Archaeozoological studies, A.T. Clason éd., Groningen 1975, 7-17, 8 fig.

Mais la mise en œuvre d'un tamisage reste à envisager au terme d'un compromis entre une amélioration des résultats qu'on peut obtenir et les conditions de fouille qui permettent ou non de mener à bien cette opération (sauvetage, fouille programmée, ...). La taille de l'échantillon traité reste à déterminer en fonction du résultat souhaité et des impératifs matériels de la fouille.

\section{PREPARATION dU MATÉRIEL OSSEUX}

Le nettoyage : il doit tenir compte de deux préoccupations : livrer au paléozoologue un matériel propre et directement accessible à l'étude ; ménager les pièces fragiles et, dans tous les cas, les surfaces osseuses dont l'examen est d'un grand intérêt (boucherie, industrie, utilisation de l'os, corrosion). Ces deux impératifs sont parfois contradictoires.

Dans le cas d'un matériel en bon état, la technique est la même que pour la céramique (eau et brosse douce). Le séchage doit être progressif (pas en plein soleil) pour éviter l'éclatement des pièces, surtout les dents dont l'émail est très sensible aux brusques variations thermiques et hygrométriques.

Si le matériel est trop fragile, on peut se limiter à un brossage à sec, en douceur. Cependant, dans certains cas, ce nettoyage peut se révéler insuffisant et risquer de rayer la surface de l'os. Brosser l'objet à l'acétone a tous les avantages d'un lavage à l'eau (la gangue se délite et part au brossage) sans les inconvénients : le temps de séchage est de 2 à 3 minutes si la pièce a été mouillée à cœur.

Le marquage : chaque pièce doit être marquée individuellement. Dans le cas contraire, l'archéozoologue risquerait de perdre les références spatiales en regroupant les os par espèces.

Le marquage, toujours effectué sur une surface parfaitement nettoyée, doit être le plus complet possible et permettre de retrouver rapidement, à partir de la documentation (voir plus bas) le site, la structure, le secteur et la couche d'origine, ainsi que la position exacte de chaque pièce, lorsque les conditions de fouille ont permis ce dernier relevé. N'oublions pas, en outre, que les clichés de certaines pièces osseuses méritent, autant que la céramique, de figurer dans les publications. Le marquage devra donc être discret.

L'utilisation du vernis sur les inscriptions est délicate. Il faut en effet éviter qu'il ne se décolle, emportant avec lui l'information. II est donc recommandé, si l'on veut employer ce procédé, d'utiliser un vernis très fluide qui, en pénétrant, "enracinera" l'inscription dans l'os. Ici encore, la technique est à adapter à chaque matériel.

Signalons qu'un des signataires de l'article (L.J.) consolide les ossements à l'acétate de cellulose et utilise une colle cellulosique, deux produits solubles dans l'acétone et dont les actions se combinent et se complètent. Pour protéger les marquages, il se sert d'un vernis traditionnel d'ébéniste, soluble uniquement à l'alcool. L'expérience montre que des pièces marquées et vernies depuis plus de dix ans et manipulées constamment, sont toujours parfaitement lisibles et que le vernis résiste à des immersions prolongées dans l'acétone.

Le stockage : divers emballages sont possibles en fonction de l'état du matériel et des moyens financiers. Un seul est à proscrire dans tous les cas : la feuille d'aluminium qui s'écrase sur l'os, l'isole mal et devient très rapidement difficile à développer sans en abîmer le contenu.

De manière générale, l'emballage doit autoriser une petite circulation d'air au contact des os pour éviter l'accumulation d'humidité ou leur trop grand dessèchement. Mais ceci est à adapter à chaque cas.

L'ouverture des sachets et des boîtes est une tâche fastidieuse qu'on peut réduire au minimum en rangeant, autant que possible, les os de même origine dans le même emballage ... et en évitant de fermer les sachets plastiques à l'aide d'un nœud trop serré !

\section{La livraison du matériel :}

- Sauf accord préalable, le paléozoologue considère que les ossements qui lui parviennent représentent la totalité du matériel issu de la fouille. Le petit colis oublié qui arrive le jour où l'on termine la rédaction du manuscrit n'est pas rare. II est la cause d'une perte de temps inutile ... et d'une certaine mauvaise humeur quand il faut reprendre toutes les évaluations de Nombres Minimaux d'Individus !

L'industrie osseuse, même celle provenant de fouilles anciennes, doit accompagner l'envoi. Elle permet parfois de compléter le spectre faunique avec des espèces qui n'apparaissent pas dans les déchets alimentaires (le cerf par exemple). Elle peut aussi expliquer l'absence systématique d'une pièce anatomique.

- L'étude du matériel ne peut commencer que si le paléozoologue a tous les documents en main. 
Pour comprendre le marquage, il faut joindre aux ossements les plans avec la nomenclature des structures et des secteurs, la stratigraphie avec les calages chronologiques relatifs et absolus. L'envoi doit s'accompagner des extraits des carnets de fouille et des relevés afin que le paléozoologue qui se penche sur les remontages et les répartitions au sol puisse contribuer à l'élaboration de la synthèse paléoethnographique du site (modifier ou préciser l'interprétation).

L'idéal est de disposer, en outre, des rapports de fouilles et d'une sélection de photographies qui seront éventuellement complétés par un bref exposé des premières hypothèses d'interprétation.

II est aussi souvent utile de communiquer à l'ostéologue qui n'a pas pu se déplacer sur le chantier, les conditions de fouille et la composition de l'environnement du site (marais, rivière, forêt, falaises, ...). Cette dernière facilitera la détermination de l'avifaune, de I'herpeto-faune et des petits mammifères.

Cette documentation, doublée d'échanges oraux avec les différents membres de l'équipe qui travaillent sur le site, évitera les regrettables contradictions ou redondances qu'on trcuve encore trop souvent entre l'article du paléozoologue et celui de l'archéologue. On imagine sans mal l'intérêt de ce dialogue dans l'élaboration du résultat final.

\section{COLlaboration ENTRE ARChÉologue ET ARCHÉOZOOLOGUE}

Pour le responsable de chantier, il est devenu nécessaire d'acquérir une formation élémentaire en archéozoologie, comme en céramologie, en stratigraphie,... II doit d'autre part s'attacher à sensibiliser tous les membres de l'équipe de fouille. Par de rapides et simples exposés, il est aisé de faire comprendre que des fragments de céramique, de métal, de charbon de bois, etc., et d'os, méritent tous la même attention sur le terrain et lors des étapes ultérieures du traitement du matériel.

En effet, il n'existera jamais sur chaque site archéologique (sur chaque sauvetage!) un archéozoologue prêt à dégager tous les ossements, prêt à apporter son conseil aux multiples coins du site, à tout emporter chez lui, et à rapporter le lendemain des trésors de tableaux magiques et d'interprétations étonnantes.

Les rapports doivent être envisagés en terme de collaboration :

- I'archéozoologue doit, dans la mesure du possible, se déplacer sur le terrain et de toutes façons se documenter sur l'ensemble du site. Il se doit de répondre le plus rapidement possible aux questions que lui pose l'archéologue ou tout autre membre de l'équipe de recherche. II participera à l'élaboration de la problématique du site.

- l'archéologue doit fournir toutes les informations disponibles sur le site, et doit s'efforcer d'intégrer la contribution archéozoologique à la publication. En effet des publications comprenant des parties rédigées en commun, et des analyses spécialisées, sont beaucoup plus efficaces que des tableaux en annexe qui n'intéressent, dans le meilleur des cas, que quelques spécialistes, et qui ne sont, la plupart du temps, pas utilisés dans l'interprétation finale du site.

\section{POURQUOI CES CONTRAINTES ?}

Les ossements animaux prélevés en fouilles peuvent fournir des renseignements de trois ordres: zoologique, archéologique et ethnographique. Mais l'importance et la qualité de ces renseignements dépendent des conditions de fouilles (précision technique, problématique adoptée, temps et moyens disponibles) et de l'aléa qu'offre toujours un gisement en ce qui concerne la conservation des vestiges et des structures.

Les ossements animaux donnent, au minimum, des indications d'ordre zoologique: détermination des espèces, proportion de chacune d'elles, dénombrement des individus, proportion d'animaux chassés, âges d'abattage, taille et race des animaux, etc. Mais la précision et la fiabilité de ces indications dépendent de l'état de conservation des éléments osseux considérés, et surtout de leur nombre, en fait, de la représentativité statistique.

Pour être représentatifs d'un gisement, les éléments osseux doivent être prélevés de manière exhaustive. Pour les très grands sites, surtout s'il s'agit de fouilles de sauvetage, cette opération est difficilement réalisable. Le prélèvement doit alors constituer un échantillon vrai, c'est-à-dire réellement aléatoire, non subjectif et en fonction d'une "maille" adaptée. On ne disposera des données nécessaires qu'après une étude approfondie et conséquente d'une partie du site et de son matériel osseux.

Quel que soit le cas, le tamisage ne doit pas être exclu, si l'on veut pouvoir parler de l'ensemble de la faune et en tirer tous les renseignements possibles.

Les vestiges osseux peuvent fournir des données d'ordre archéologique. Pour qu'elles soient perceptibles et exploitables, il importe que les prélèvements mais aussi les relevés soient rigoureux (localisation de l'objet selon trois axes, notation de son pendage et de son orientation, ....). Ceci permet d'élaborer des plans de répartition et de remontage, d'étudier les concentrations des os et leur disposition en fonction de leur taille, de leur forme, et de souligner les pendages et les orientations du remplissage.

L'étude de la conservation différentielle d'une couche à une autre, de l'hétérogénéité du matériel osseux, des différences de couleur et de texture, du degré de corrosion, etc., permettent de fournir des indications sur le mode de dépôt de la couche archéologique. Ce travail nécessite que les vestiges aient été relevés par couche.

Bien entendu c'est à l'archéologue de choisir 's'il va ou non investir dans un relevé minutieux, en fonction des conditions de fouilles, de la problématique de recherche, et de la nature du gisement.

Enfin, les vestiges osseux peuvent donner matière à des interprétations d'ordre ethnographique. Les données zoologiques (proportions entre espèces, courbes d'âges, faciès reconstitués des troupeaux) permettent de se faire une idée sur l'économie de la société étudiée, voire sur ses structures sociales.

L'intérêt ethnographique des pièces trouvées en connexion, des ossements relevant de la catégorie des détritus en place, de ceux dont le contexte indique qu'il s'agit de dépôt votif ou de sacrifice animal, n'est plus à démontrer. 
Les éléments osseux peuvent aussi fournir d'eux mêmes des données d'ordre ethnographique lorsqu'ils comportent des traces de débitage, de décarnisation, d'utilisation ou de façonnage.

Les vestiges osseux peuvent donc être riches de renseignements, par eux-mêmes, par l'étude zoologique, par le contexte archéologique. Les trois domaines considérés ici n'étant pas cloisonnés, pas même hiérarchisés, mais plutôt constamment interférents (voir le tab. II).

\section{CONCLUSION}

Dans l'exposé qui précède nous avons énuméré les nécessités immédiates quant au traitement des ossements, en vue de donner à leur étude des chances d'aboutir. Mais ces quelques recettes ne sont pas suffisantes si elles ne sont pas accompagnées d'une étroite collaboration entre l'archéozoologue et l'archéologue. Ce dernier a une vision générale de la problématique d'un site et doit y associer l'archéozoologue, du terrain jusqu'à la publication. C'est à cette condition qu'on obtiendra les meilleurs résultats.
Malheureusement il n'existe en France qu'une trentaine de chercheurs dans ce domaine, dont une majorité de bénévoles. La création de postes d'archéozoologues est une nécessité, comme l'est celle de postes de palynologues, d'anthracologues, de géologues, ..

Le problème mérite d'être examiné au regard des questions de l'ensemble de l'archéologie française, et il ne sera le plus justement résolu que dans ce cadre.

Mais il appartient aux archéozoologues de donner leur avis, d'avancer leurs arguments, pour mieux conseiller les instances de décisions, pour faciliter leurs choix, pour mieux proposer une programmation de ces créations de postes.

\section{BIBLIOGRAPHIE SUCCINTE}

POULAIN, T., 1976. L'étude des ossements animaux et son apport à l'archéologie. Centre de recherche sur les techniques gréco romaines, $n^{\circ} 6$, Université de Dijon, 131 p., nombreuses figures, $5 \mathrm{PI}$.

SCHMID, E., 1972. Atlas of animal bones. Tierknochenatlas, Elsevier Publishing Company, Amsterdam, 1972, 153 p., 39 figures $37 \mathrm{PI}$.

Tableau I

DE LA FOUILLE DES OSSEMENTS ANIMAUX

IL FAUT :

- Tout prélever

(éviter les pertes sur la fouille,

et après celle-ci).

- Préserver la surface des os.

- Eviter les fractures.

- Relever les connexions entre os.

- Ménager les pièces importantes,

et les crânes en particulier.

- Faire un relevé adapté.
POUR CELA, ON PEUT :

- Tamiser, ce qui augmente

le nombre de restes et la liste des

des espèces (microfaune).

- Utiliser des outils en bois.

- Consolider les os avant le

"démontage".

- Les fouiller complètement avant

de les démonter.

- Les démonter sans les briser et les transporter de même.

- Les enregistrer par structures, par ensembles stratigraphiques, par carré, par vestiges sur plan ou sur photo.
CEPENDANT :

- le tamisage prend du temps,

et les résultats ne sont pas garantis.

- Il est toutefois possible de bien fouiller avec d'autres instruments.

- Les cas où la consolidation est nécessaire restent rares.

- Cela n'est pas toujours aisé. II convient alors de contacter un spécialiste.

- La restauration d'un crâne brisé est envisageable, mais elle est longue (des dizaines d'heures)

- Trop de précisions ne nuit pas à l'étude.

Tableau II

\section{CONTRAINTES}

Ramassage systématique/tamisage.

Préservation des surfaces osseuses

(fouille, nettoyage, stockage).

Repérage et enregistrement des connexions.
MÉTHODES D'ÉTUDE

Détermination spécifique.

Spectre faunique.

Biométrie et morphologie

(crâne, chevilles osseuses, ...).

Caractéristique des animaux :

åge, sexe, taille, poids,

Représentation relative des

différentes parties du squelette.

Traces : découpe, corrosion, chien, rongeur, utilisation.

Remontage (collage, connexion, ...) et répartitions.

\section{RÉSULTATS POSSIBLES}

Evaluation optimale de l'importance relative des espèces (NR, NMI, NEI) Chasse/Elevage milieu environnant

Détermination subspécifique et raciale.

Caractéristiques de l'élevage - buts,

évolution, comparaison.

Techniques bouchères.

Industrie de l'os

Gestion du troupeau.

Taphonomı.

Contribution à l'interprétation de la dynamique du dépôt et de la nature du site. 